\title{
Energy Efficient Routing Protocol for Wireless Sensor Network
}

\author{
Noor Zaman*, Tung Jang Low**, Turki Alghamdi*** \\ *** Department of Computer Science \& Information Science, Universiti Tecknologi PETRONAS, Malaysia, ***College of \\ Computer and Information System, Umm Al-Qura University, Saudi Arabia
}

Noorzaman650@hotmail.com, lowtanjung@petronas.com.my, taghamdi@uqu.edu.sa

\begin{abstract}
Nowadays Wireless Sensor Networks WSNs are playing a vital role in several application areas ranging health to battle field. Wireless sensor networks are easy to deploy due to its unique characteristics of size and self-organizing networks. Wireless sensor nodes contain small unchangeable and not chargeable batteries. It is a resource constraint type network. Routing in WSN is most expensive task as it utilizes more power resources. This paper is intended to introduce energy efficient routing protocol, known as Position Responsive Routing Protocol (PRRP) to enhance energy efficiency of WSN. Position responsive routing protocol differs in several ways than other existing routing techniques. Position response routing protocol approach allows fair distribution of gatewaylcluster head selection, maximum possible distance minimization among nodes and gateways \cluster heads to utilize less energy. Position responsive routing protocol shows significant improvement of $45 \%$ in energy efficiency of wireless sensor network life time as a whole by increasing battery life of individual nodes. Furthermore PRRP shows drastic increases for data throughput and provide better solution to routing energy hole due to it fair distributed approach of gateway selection.
\end{abstract}

Keywords - WSN, Energy efficient, PRRP position responsive routing protocol, CELRP, Routing protocol,

\section{INTRODUCTION}

WSN is a popular and have capability to high penetrate with several applications areas. It consists of small nodes having limited sensing, computation, and wireless communications capabilities [1-2]. Sensor nodes normally sensed data and forward sensed data to the base station such as temperature, sound, vibration, pressure, motion or pollutants [3]. Sensor nodes are resource constraint type of network and contain very tiny size of irreplaceable and not chargeable batteries. Network life is the highly concern, energy become reason to dyes of nodes and network partially fully stop working [4-6]. Energy consumed more during path finding and data transmission operations terms as routing. Routing is the most challenging issue and direct concern to energy in WSN comparable with ad hoc and cellular network [7-8]. Clustering technique for routing in WSN is considered most suited based on its characteristics such as energy-efficient, scalable, lower latency, etc. In clustering, WSN network is divided into sub networkslclusters and each cluster has cluster head which is responsible to collect the sensed data from his cluster and forward it to the base station [9]. Cluster heads consumed more energy due to collecting and forwarding data from cluster while remaining nodes in the clusters still have more energy of $90 \%$ of their initial energy [10-12]. This situation normally happened due to unbalanced energy assumption which causes more drain of energy from nodes far from cluster heads [13] in random fashion from sensor nodes. To address this issue, several energy efficient routing algorithms and protocols have been propose recently, including cluster based protocols [14-19], power-aware routing [20-22] and multi-level transmission radii routing [23]. The minimum energy routing problem has been addressed in [24]. If sensor nodes consume energy more equitably, they continue to provide connectivity for longer and the network lifetime increases. [25-35].

As sensor networks have specific requirements on energy saving, data-oriented communication, and interconnection between non-IP and IP, therefore sensor network-dedicated routing protocols may be required, for Energy efficient routing scheme.

\section{Problem Statement}

Sensor network has primary role to sense and forward data to destination or base station BS, resulting of any physical event occurrence. Routing plays a key role to identify path and transfer data in energy constraint sensor network. Initially routs defined by the nodes then nodes become able to send or receive the data by using those routing paths. In case if sensed data is available to some segments of network but network not able to transfer it to the destination due to the energy deplete of sensor nodes for some segments.

\section{RELATED WORK}

Several routing protocols have been developed recently to address the energy efficiency issue. WSNs routing protocols normally specified in following types.

1. Flat routing Protocols

2. Hierarchal routing Protocols

3. Location based routing Protocols 
Hierarchal routing protocols work in cluster formation and considered to be more energy efficient due to their unique characteristics. Recently proposed Cluster based Energy Efficient Location Routing Protocol (CELRP), also belongs to Hierarchal type. In CELRP sensor nodes are normally distributed into clusters and divided into different quadrants. Each quadrant contains two clustering and sensor nodes that transmit data with two hops data transmission [36]. $\mathrm{CH}$ is selected based on the node with maximum residual energy and minimum distance to the base station in each cluster. While the $\mathrm{CH}$ which has the highest energy residual is chosen as the $\mathrm{CH}$ Leader between all the other CHs. CELRP applies Greedy algorithm among cluster heads and forward data to the sink. The collection works as nodes sends data to $\mathrm{CH}$ and then $\mathrm{CH}$ forward their data to the sink through $\mathrm{CH}$ leader by minimizing number of hops. CELRP assumed that the Base Station has all the information including the sensor nodes, the residual energy and the distance of node from sink. Sink is place far from the sensor node area. The CELRP based on three phases, in first phase it works for the formation of clusters, network divided into four quadrants and then it forms clusters. In second phase it selects $\mathrm{CH}$ and $\mathrm{CH}$ leader on the basis of its energy and finally with third phase it transfer data to the sink. CELRP has main limitations like its number of children nodes is high in the clusters and secondly its CHleader choosing mechanism works on the basis of energy level, in most of the cases CHleader is not the closest to $\mathrm{CH}$, hence it causes more energy drain because of longer distance. At the same time it uses Greedy approach for data transmission which also causes loss of energy efficiency.

Hence it is highly needed to design an energy efficient routing protocol with assumptions closer to the real, we are position responsive routing protocol (PRRP) WSN routing protocol which is more energy efficient than the existing protocols.

\section{SySTEM MODEL}

Our assumptions for sensor network are such that, sensor nodes are randomly distributed over an area of $90 \mathrm{x}$ 90 meters with following network properties.

1. Network is static and nodes are distributed in random format, while area is divided in equal square grid format.

2. There is exists only one base station, which is deployed in the center of the area.

3. The energy of sensor nodes cannot be recharged.

4. Sensor nodes are location aware.

5. The radio power can be controlled, i.e., a node can vary its transmission power.

Above all assumption are on wide scope, assumption no. 5 , is becoming the cause of energy saving, as nodes will be aware about their location and sink too, hence the amount of energy which normally network always use to find out the initial location will be saved.
Concerning energy efficiency of PRRP, This section presents an analysis of energy utilization in terms of energy consumed by cluster head nodes and leaf nodes. Consider a wireless sensor network comprising $N$ wireless sensor nodes that are uniformly distributed within a grid of size $\mathrm{m}$ $\times \mathrm{m}$; within each cell one of node acts as the cluster head $(\mathrm{CH})$, so the number of cluster heads will be same as the number of cells, i.e. $m^{2}$. We shall use the following symbols to denote quantities mentioned against each:

$k$ : Number of leaf nodes in a cell; i.e. $\mathrm{k}=\frac{N}{m^{2}}-1$; $($ not counting the $\mathrm{CH}$ ),

$E_{t}$ : Energy to transmit one sample,

$E_{r} \quad:$ Energy to receive one sample,

$E_{\text {in }}:$ Initial energy provided to each node at the time of deployment,

$E_{\text {th }}$ : Energy Threshold below which a node cannot act as a cluster head

$E_{l n}:$ Energy consumed by Leaf node

$P_{s}$ : Power consumed while a node is asleep,

$T_{t}$ : Time to transmit one sample,

$T_{s} \quad$ : Sampling interval,

\section{Energy Consumed by leaf nodes}

For leaf nodes, there are two modes of energy consumption namely: energy consumed in sleep state and energy consumed during transmission. During each sampling interval $T_{s}$, a leaf node spends $T_{t}$ time for transmission and the remaining $T_{s}-T_{t}$ time in sleep mode. Thus energy consumed by a leaf node, $E_{l n}$ can be calculated as:

$$
E_{l n}=E_{t}+\left(T_{s}-T_{t}\right) P_{s}
$$

\section{Energy Consumed by cluster heads}

As cluster head spends its life in three modes, namely: receiving samples from leaf nodes, transmitting its data and sleep mode. Since, the energy consumed to receive a sample is $E_{r}$ and there are ${ }^{k}$ leaf nodes in each cell; thus, total energy consumed by $\mathrm{CH}$ in receiving $k$ samples is $k E_{r}$, while energy consumed by $\mathrm{CH}$ during transmission is $E_{t}$. During each sampling interval, a $\mathrm{CH}$ spends $k T_{t}$ time for 
receiving samples, $T_{t}$ time for transmission and remaining $T_{s}-(k+1) T_{t} \quad$ time in sleep mode; thus energy consumed in sleep mode is $P_{s} \times\left(T_{s}-(k+1) T_{t}\right)$. The total energy consumed by a $\mathrm{CH}$ during one sampling interval, $E_{c h}$ is given by:

$$
E_{c h}=E_{t}+k E_{r}+\left(T_{s}-(k+1) T_{t}\right) P_{s}
$$

Next, we calculate life of a $\mathrm{CH}$ in terms of number of sampling intervals, $N_{s}$ while its remaining energy is more than the threshold $E_{t h}$. Thus,

Total life of a CH, $\quad N_{s}=\frac{E_{\text {in }}-E_{\text {th }}}{E_{t}+k E_{Y}+\left(T_{s}-(k+1) T_{t}\right) P_{s}} \quad(\mathrm{C})$

\section{Life of a leaf node}

Once a $\mathrm{CH}$ dies, a new round of cluster head selection is initiated. In the following paragraphs, we show that leaf nodes still have enough energy left to participate as cluster heads in future rounds. The initial round leading to exhaustion of a $\mathrm{CH}$ consists of $N_{s}$ sampling intervals as shown above. Therefore, energy consumed by a leaf node during the first round, $E_{l n 1}$, is given by $N_{s} \times E_{l n}$; i.e.,

$$
E_{l n 1}=N_{s} \times E_{l n}
$$

Using Eqs A and C, Eq D becomes:

$$
E_{l n 1}=\frac{E_{\text {in }}-E_{\mathrm{th}}}{E_{\mathrm{t}}+k E_{\mathrm{r}}+\left(T_{s}-(k+1) T_{\mathrm{t}}\right) P_{s}} \star\left(E_{t}+\left(T_{s}-T_{t}\right) P_{s}\right)
$$

Assume that $P_{s} \cong 0$ and divide equation D with $E_{t}$ we will get

$$
E_{l n 1} \cong \frac{E_{t}\left(E_{i n-} E_{t h}\right)}{1+k E_{r} / E_{t}}
$$

Typically, energy consumed during transmission of a number of bits is about four times as much as energy consumed for receiving the same number of bits[37], that is to say that $E_{t}=4 E_{r}$.

$$
\text { So } \quad E_{\ln 1} \cong \frac{E_{\text {in }-E_{\text {th }}}}{1+k / 4}
$$

It is obvious from Equation (E) that at the end of first round, for a sufficiently large cluster size, leaf nodes consume only a fraction of their energy reserve. Thus, the network will last for many rounds after the death of first cluster head.

\section{RESULTS \& DISCUSSION}

In this section, it is attempted to compare our proposed PRRP protocol with CELRP. In CELRP sensor nodes are normally distributed into clusters and divided into different quadrants. Each quadrant contains two clustering and sensor nodes that transmit data with two hops data transmission [36]. $\mathrm{CH}$ is selected based on the node with maximum residual energy and minimum distance to the base station in each cluster. While the $\mathrm{CH}$ which has the highest energy residual is chosen as the $\mathrm{CH}$ Leader between all the other CHs. CELRP applies Greedy algorithm among cluster heads and forward data to the sink. The collection works as nodes sends data to $\mathrm{CH}$ and then $\mathrm{CH}$ forward their data to the sink through $\mathrm{CH}$ leader by minimizing number of hops. CELRP assumed that the Base Station has all the information including the sensor nodes, the residual energy and the distance of node from sink. Sink is place far from the sensor node area.

CELRP have main limitations such as the number of children nodes is higher in the clusters like LEACH and secondly its CHleader choosing mechanism works on the basis of energy level, in most of the cases CHleader is not the closest to CHleader, hence it causes more energy drain due to longer distance. CELRP consider the placement of sink far from the sensing area and at the same time it uses Greedy approach for data transmission which both also caused loss of energy efficiency. Hence above stated limitations causes major sources of energy drain. PRRP becomes more energy efficient by using different techniques for stated limitations besides of other considerations. This section is aimed to discuss the performance evaluation of PRRP and CELRP and to compare them on the basis of number of live nodes vs time, total consumed energy and network throughput one by one and collectively for different data transmission periods.

\section{A: Comparison Analysis on the basis of Live Nodes}

This section describes comparison analysis of results for live nodes among CELRP and CELRP for different data transmission periods individually and collectively. Figure 01 shows a comparison between PRRP and CELRP in terms of number of live nodes initially for single period of transmission. Here, our network is tested with different periods and rounds of data transmission such as 1,5 and 10 . After discussing all one by one, all of them finally and collectively shows for a better understanding of the difference between CELRP and PRRP. The following Figure 01 presents the comparison for the single round only.

Our observation shows significant results of PRRP over CELRP for different periods and rounds. Initially its result shows little differences but finally through the increase in 
the number of the periods, it brings more significant differences as shown in Figure 01. PRRP shows a more time network operational with single period, which is almost up to 140 seconds, yet CELRP goes up to 120 seconds only.

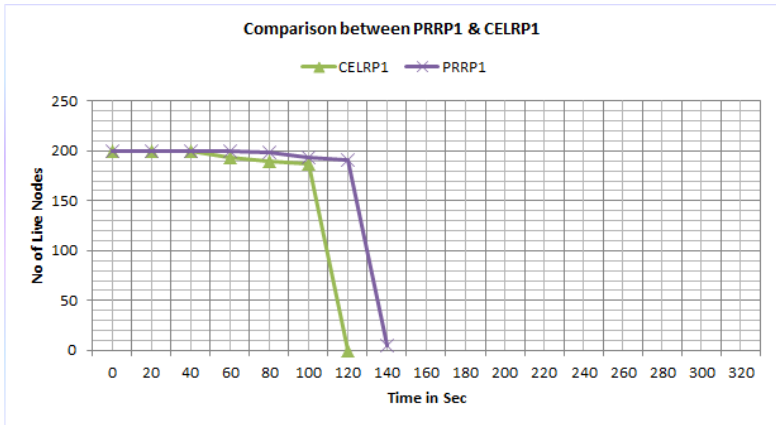

Figure 01: Number of Live Nodes vs. Time, (PRRP \& CELRP with one Period)

Furthermore, we observe the same difference in a notable improvement when testing our network for the 5 periods as shown in Figure 02 below. The results then show that PRRP goes up 300 seconds of time, while CELRP reaches up to 200 seconds. This difference will increase whenever we test the network for the different and more number of rounds. This positive difference comes due to the efficient mechanism of the gateways selection and functioning in PRRP. The area of operation of the neighbors of the PRRP is always close to its gateway for saving its energy level as shown in several different cases of different rounds of data transmissions and at the same time $\mathrm{CH}$ have less distance from the sink as it is placed in the center.

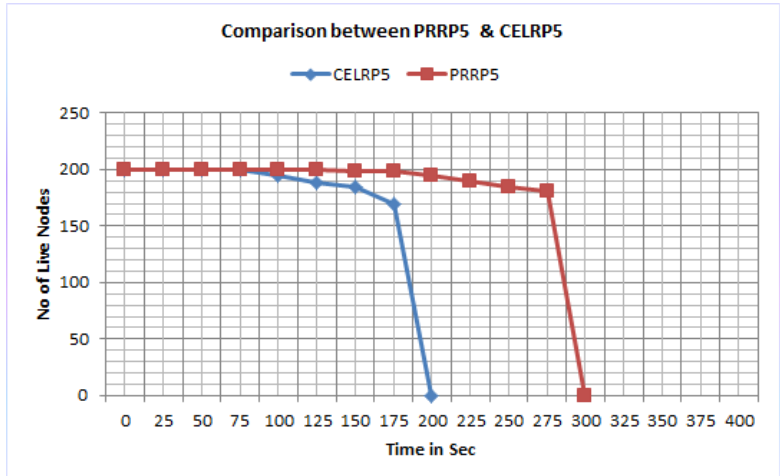

Figure 02: Number of Live Nodes vs. Time, (PRRP \& CELRP with Five Periods)

In the third test, we compare CELRP and PRRP for 10 numbers of periods as shown in Figure 03. It shows that PRRP has a more significant performance than CELRP for all scenarios. For example, considering the scenario of 10 data transmission periods, the network life is significant improved for PRRP with an excellent improvement in sensor network lifetime, which is almost 400 seconds, while for LEACH it is about 300 seconds. The improvement in PRRP is because of the efficient use of the energy for all nodes is up to its last limit and also has an opportunity to participate all nodes in the network without having any network isolated part with it. Secondly, the energy enhancement in PRRP is because of minimizeed energy consumption with each node by decreasing the distance between the gateway and the non-leaf nodes and between the sink at the same time.

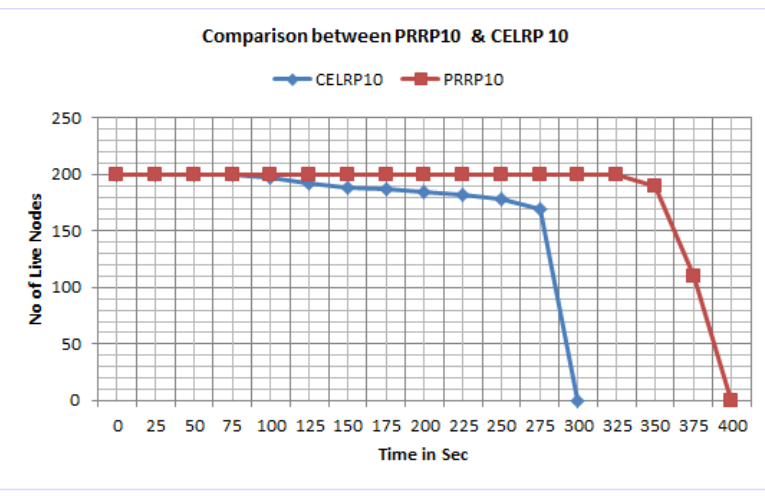

Figure 03: Number of Live Nodes vs. Time, (PRRP \& CELRP with Ten Period)

By comparing the energy consumed by a node in CELRP and PRRP, a difference in status of the nodes will be found in CELRP and PRRP. It should be considered that there are three different types of the nodes in PRRP; while in CELRP it is only two types of nodes such as normal node or non-head node and Cluster head or head node, while the $\mathrm{CH}$ leader is to be chosen from one of the $\mathrm{CH}$ on the basis of its energy level. In PRRP nodes on the other hand has three following different types such as non-leaf node, leaf node and gateway nodes. Energy can be compared on the basis of energy consumed through all nodes during transmitting, receiving and idle-listening state.

When we compare PRRP with CELRP for different periods of data transmission, PRRP always shows an excellent improvement with energy saving of the network, as collectively shown in Figure 04. Simulation results shows a significant result with an increase in number of the rounds of PRRP, as PRRP uses a mechanism in which if the same node has some residual energy level, each node can be a gateway until the end. This approach enables PRRP to save more energy and use all nodes energy up to its last maximum limit. The difference can be easily monitored in Figure 04 presenting the collective results of different data transmission rounds. 


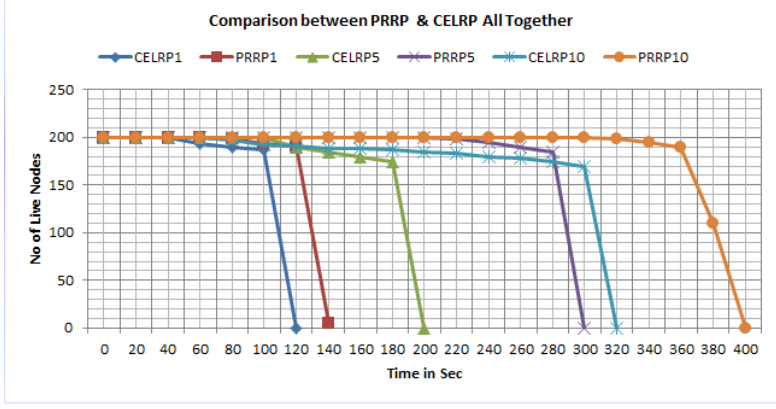

Figure 04: Number of Live Nodes vs. Time, (PRRP \& CELRP Collectively)

\section{B: Comparison Analysis on the basis of Total Energy Consumed}

As mentioned in earlier sections, in tree building phase in PRRP, the nodes can transmit signals with minimum energy level. Whilst, the parent can select node which is closer to the node on very short distance which saves its energy level. In comparison with CELRP, parent of node can be any node, CELRP do not consider the distance among nodes and $\mathrm{CHs}$. If we compare the energy consumption by leaf node in PRRP and normal node in CELRP, it can be noted that both types of nodes will not lose energy due to receiving data or idle listening. As a TDMA transmission scheme is implemented, they will not lose any energy and node will be only $O N$ at its schedule time only.

The transmission distance is very short in PRRP, as the parent node is near leaf node. While in CELRP the main part of the energy is consumed during the data transmission phase in particular when the normal node is at a distance from the cluster head and mostly $\mathrm{CH}$ leader is on longer distance from the $\mathrm{CH}$. The second main reason for the energy loss in CELRP is the sink is place on long distance hence overheads becomes more for nodes which are far from the sink. Thus, there is a chance that the $\mathrm{CH}$ leader and cluster head itself might be so far from the normal nodes and becomes the factor of energy loss during the data transmission phase. Furhermore, by comparing non-leaf node and the cluster head node of CELRP, it can be found that non-leaf node in PRRP is not losing its energy due to the idle listing as it schedules to receive and transmit data at the specific time slots only. It is also found that in CELRP cluster head should remain $\mathrm{ON}$ to transmit its data to the sink and cluster head uses CSMA technique. Therefore, it loses the energy.

The number of the children is less related to any gateway in PRRP. It is due to the gateway, compared to the head in CELRP, has less number of children, thus making it consume less energy in receiving data. This also comes to be a factor for a deep tree building in which neighbor nodes remain closer to the gateway. In CELRP any cluster head, conversely, might have a more number of children nodes at different distances. This in CELRP also becomes the factor of energy decrease. For this, the total consumed energy in PRRP will be smaller in comparison to CELRP as shown in the following Figure 05 for different data transmission periods.

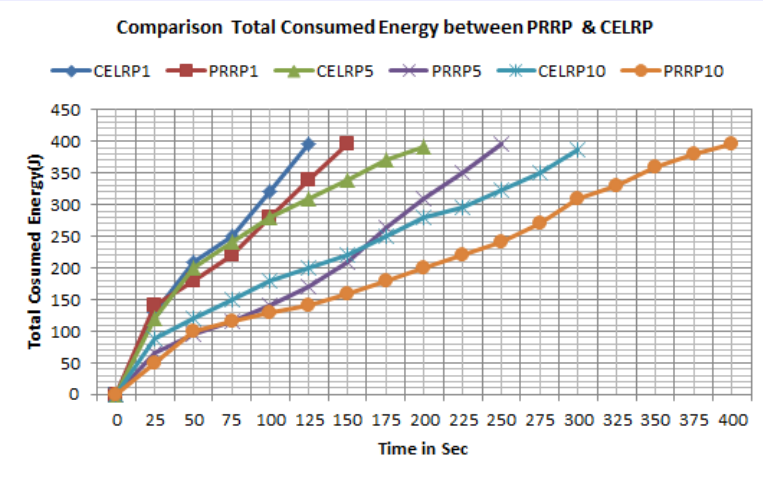

Figure 05: Total Consumed energy vs. Time, (CELRP \& PRRP)

As an illustration, if we compare to 10 data transmission periods at $\mathrm{t}=150$ seconds, the total consumed energy is about $230 \mathrm{~J}$ in CELRP and about $140 \mathrm{~J}$ in PRRP. The consumed energy is dropped by a significant difference almost $100 \%$. The overall energy consumed by the end of the network lifetime is efficient by both the protocols. However, the important concern is that which protocol can achieve a longer lifetime and higher throughput for the same initial energy level.

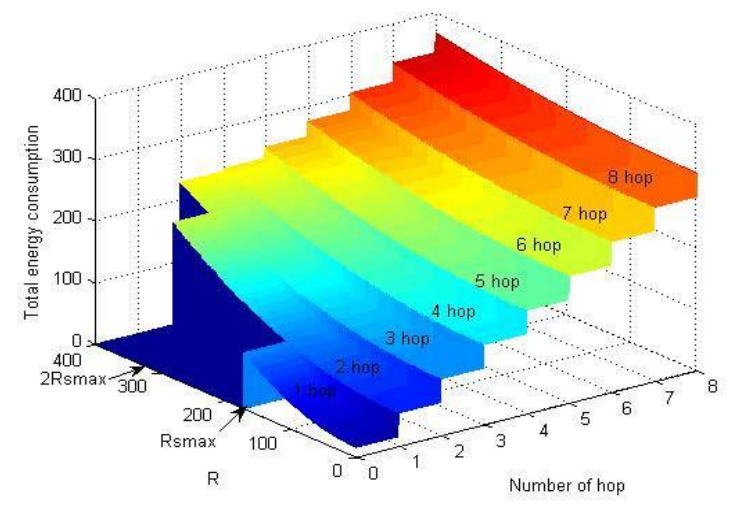

Figure 06: Energy Consumptions over the number of hop.

Figure 06 shows total energy consumption based on simulations for different hops, where the number of nodes were considered as of 200 with having energy level of $2 \mathrm{~J}$ for each and overall energy of $400 \mathrm{~J}$. The author has shown one more aspect of the result that if the sink is placed far from the sensing field, in that case the number of hopes will increase from the node to the sink that increases the loss of energy as distance increases from the node to the sink. In PRRP, the sink is placed in the center of the sensing field to decrease the possible number of hops among nodes and the 
sink and at the same time to decrease the distance among the nodes and $\mathrm{CHs}$.

\section{C: Comparison Analysis on the basis Throughput}

This sub section describes about network throughput which shows a substantial growth because of increase in overall network energy efficiency. In PRRP protocol, the main energy is saved during the data transmission phase with multiple data transmission periods by utilizing same build tree for multiple periods. The network survives longer because of less distance between nodes and gateways. The following Table 01 is showing the comparison between PRRP and CELRP protocols in term of the data packets delivery to the sink for different periods.

Table 01: A comparison between PRRP and CELRP in terms of throughput

\begin{tabular}{|c|c|c|c|}
\hline Periods & PRRP & CELRP & $\begin{array}{l}\text { Improvement } \\
\text { with PRRP }\end{array}$ \\
\hline $\begin{array}{c}\text { 1 Data } \\
\text { Transmission } \\
\text { period }\end{array}$ & 16733 & 5950 & $\begin{array}{c}2.81 \\
\text { times }\end{array}$ \\
\hline $\begin{array}{c}\text { 5 Data } \\
\text { Transmission } \\
\text { periods }\end{array}$ & 54555 & 19985 & $\begin{array}{c}2.72 \\
\text { times }\end{array}$ \\
\hline $\begin{array}{c}\text { 10 Data } \\
\text { Transmission } \\
\text { periods }\end{array}$ & 65305 & 35710 & $\begin{array}{c}1.82 \\
\text { times }\end{array}$ \\
\hline
\end{tabular}

Overall, the performance of the PRRP is more significant than the one in CELRP. PRRP, in this case by enhancing the life of the sensor nodes and by utilizing each sensor nodes energy resource up to the maximum level, enhances the overall life time and energy efficiency of the network. As seen in Table 01, the comparison of PRRP and CELRP with different aspects is presented. The overall significant throughput is improved in PRRP through an application of it with multiple rounds of data transmission.

This improvement in fact is determined by two major factors. The first factor is related to the increase in the network lifetime in PRRP entirely that ensures more influx of data packet, directly giving an effect on the throughput of the network. The second one is about our assumption that any node in PRRP can become a gateway on the basis of different parameters, allowing the maximum nodes to be the gateways. However, gateway selection will be based on different parameters including energy efficiency, Position from the sink and number of neighboring nodes. Hence, the increase in number of gateways based on stated parameters in fact, comparable with CELRP, can decrease the depth of the tree, and also cause a good impact for the throughput of the network. This increase of data throughput is directly linked with overall energy efficiency of sensor network which can be achieved through our proposed PRRP.

\section{CONCLUSION}

Wireless sensor network has important role and use, due to it's diversify approach and range of applications. WSN is the only most suitable and easy way of deployment in remote and hard areas. Routing is the main expensive operation for nodes energy consumption. This paper proposed new routing protocol known as Position Responsive Routing Protocol PRRP. Our proposed PRRP addresses energy efficiency, data throughput and routing hole under certain controlled conditions. Our simulation based research results showed a significant energy efficiency improvement of $35 \%$ to $45 \%$ in WSNs by increasing overall energy efficiency and life time. PRRP also shows a significant improvement of data throughput approximately 3 times to existence protocol CELRP. PRRP also addresses routing hole issue due to distribution approach of gateway selection and providing chance to maximum nodes for data transmission.

\section{REFERENCES:}

[1] Jamal N. Al-Karaki, Ahmed E Kamal, "Routing Techniques in Wireless Sensor Network: A Survey", IEEE Wireless Communication December 2004.

[2] Noor Zaman and A. Abdullah, "Energy Efficient Routing in Wireless Sensor Network: Research Issues and Challenges" in IEEE International Conference on Intelligence and Information Technology, ICIIT 2010. pp. 239-243

[3] I.F. Akyildiz, W. Su, Y. Sankarasubramaniam, and E. Cayirci, "A Survey on Sensor Network", IEEE Communication Magazine, vol. 40, no.8, pp. 102-116, August 2002.

[4] Akyildiz, I. F., W. Su, Y. Sankarsubramaniam and E. Cayirci, A survey on sensor networks, IEEE, Communications Magazines, vol.40, no.8, pp.102-114, 2002.

[5] Bhardwaj, M. and A. Chandrakasan, Bounding the lifetime of sensor networks via optimal role, assignments, Proc. of the INFOCOM'02, pp.1587-1596, 2002

[6] Zussman, G. and A. Segall, Energy efficient routing in Ad Hoc disaster recovery networks, Proc. Of the INFOCOM'03, pp.682-691, 2003.

[7] Q. Cao, T. Abdelzaher, T. He, and R. Kravets, "Cluster-Based Forwarding for Reliable End-to-End Delivery in Wireless Sensor Networks", IEEE Infocom'07, May 2007.

[8] Charles Pandana and K. J. Ray Liu "Robust Connectivity-Aware Energy-Efficient Routing for Wireless Sensor Networks," IEEE transactions on wireless communications, vol. 7, no. 10, October 2008

[9] Younis, O.; Fahmy, S. HEED: A hybrid, energy-efficient, distributed clustering approach for ad hoc sensor networks. IEEE Trans. Mob. Comput. 2004, 3, 366-379.

[10] Lian, J.; Naik, K.; Agnew, G.B. Data capacity improvement of wireless sensor networks using non-uniform sensor distribution. Int. J. Distrib. Sens. Netw. 2006, 2, 121-145.

[11] Olariu S.; Stojmenovic, I. Design Guidelines for Maximizing Lifetime and Avoiding Energy Holes in Sensor Networks with Uniform Distribution and Uniform Reporting. In Proceedings of the 25th Annual Joint Conference of the IEEE Computer and Communications Societies (INFOCOM 2006), Barcelona, Spain, 23-29 April 2006; pp. 1-12.

[12] Wadaa, A.; Olariu, S.; Wilson, L.; Jones, K.; Eltoweissy, M. Training a wireless sensor network. Mob. Netw. Appl. 2005, 10, 151-168. 
[13] Li, X.; Nayak, A.; Stojmenovic, I. Sink mobility in wireless sensor networks. Int. Fed. Inf. Proc. 2010, 153-184.

[14] Heinzelman, W.R.; Chandrakasan, A.; Balakrishnan, H. EnergyEfficient Communication Protocol for Wireless Micro-Sensor Networks. In Proceedings of the 33rd Annual Hawaii International Conference on System Sciences (HICSS 2000), Maui, HI, USA, 4-7 January 2000; p. 10.

[15] Heinzelman, W.R.; Chandrakasan, A.; Balakrishnan, H. An application-specific protocol architecture for wireless sensor networks. IEEE Trans. Wirel. Commun. 2002, 1, 660-670.

[16] Khalil, E.A.; Attea, B.A. Energy-aware evolutionary routing protocol for dynamic clustering of wireless sensor networks. Swarm Evol. Comput. 2011, 1, 195-203.

[17] Gautam, N.; Pyun, J.Y. Distance aware intelligent clustering protocol for wireless sensor networks. J. Commun. Netw. 2010, 12, 122-129.

[18] Li, C.F.; Ye, M.; Chen, G.H.; Wu, J. An Energy-Efficient Unequal Clustering Mechanism for Wireless Sensor Networks. In Proceedings of the IEEE International Conference of Mobile Ad hoc and Sensor System, Washington, DC, USA, 7 November 2005; pp. 604-611.

[19] Yu, J.G.; Qi, Y.Y.; Wang, G.H.; Gu, X. A cluster-based routing protocol for wireless sensor networks with Nonuniform node distribution. Int. J. Electron. Commun. 2012, 66, 54-61.

[20] Buragohain, C.; Agrawal, D.; Suri, S. Power Aware Routing for Sensor Databases. In Proceedings of the 24th Annual Joint Conference of the IEEE Computer and Communications Societies (INFOCOM 2005), Miami, FL, USA, 13-17 March 2005; pp.1747-1757.

[21] Singh, S.; Woo, M.; Raghavendra, C.S. Power-Aware Routing in Mobile ad hoc Networks. In Proceedings of the $4^{\text {th }}$ Annual ACM/IEEE International Conference on Mobile Computing and Networking (MobiCom 1998), New York, NY, USA, 1998; pp.181-190.

[22] Stojmenovic, I.; Lin, X. Power aware localized routing in wireless networks. IEEE Trans. Parall. Distr. 2001, 12, 1122-1133.

[23] Li, N.; Hou, J.C. Topology Control in Heterogeneous Wireless Networks: Problems and Solutions. In Proceedings of the 23th Annual Joint Conference of the IEEE Computer and Communications Societies (INFOCOM 2004), Hong Kong, 7-11 March 2004; pp. 1735-1746.

[24] A. K. Sadek, W. Su, and K. J. R. Liu "Multinode cooperative communications in wireless networks," IEEE Trans. Signal Processing, vol. 55, no. 1, pp. 341-355, 2007.

[25] Estrin, D.; Govindan, R.; Heidemann, J. and Kumar, S., "Next Century Challenges: Scalable Coordination in Sensor Networks ," Proceedings of 5th annual IEEE/ACM international conference on Mobile Computing and Networking, pp. 263-270, 1999.

[26] Rentala, P.; Musunnuri, R.;Gandham, S. and Saxena U., "Survey on Sensor Networks ," Available online at: http://www.mdpi.net/sensors.

[27] Wood, A. D., and Stankovic, J. A., Security of Distributed, Ubiquitous, and Embedded Computing Platforms, to appear in Wiley Handbook of Science and Technology for Homeland Security, Voeller, J. G. (Ed.), John Wiley \& Sons, Hoboken, NJ, 2008.

[28] A. K. Sadek, Z. Han, and K. J. Ray Liu, "A distributed relayassignment algorithm for cooperative communications in wireless networks," in Proc. IEEE International Conference on Communications (ICC), vol. 4, pp. 1592-1597, June 2006.

[29] Chang, J. H. and L. Tassiulas, Routing for maximum system lifetime in wireless Ad Hoc networks, Proc. of the ACCCC'99, 1999.

[30] Noor Zaman and A. Abdullah, " Position Responsive Routing Protocol (PRRP)". In IEEE The $13^{\text {th }}$ International Conference on Advance Communication Technology ICACT 2011, Seoul Korea. Pp.644-648

[31] Noor Zaman, and A. Abdullah, "Energy Optimization through Position Responsive Routing Protocol (PRRP) in Wireless Sensor Network (WSN)". In International Journal of Information and Electronics Engineering IJIEE, 2012, Vol. 02, No.05, September 2012, pp. 748-751.

[32] Noor Zaman, Low Tang Jung, Fawaz Alsaade, Turki Alghamdi, "Wireless Sensor Network (WSN): Routing Security, Reliability and Energy Efficiency". In Journal of Applied Science, Science alert, Vol. 12, No.16, 2012 USA, pp. 593-597.

[33] Noor Zaman and A. Abdullah, "Different Techniques Towards Enhancing Wireless Sensor Network (WSN) Routing Energy Efficiency and Quality of Service (QoS)". in World Applied Science Journal (WASJ), Vol.13, no 4, 2011, pp. 798-805.

[34] Noor Zaman and A. Abdullah, Low Tang Jung, "Optimization of energy usage in wireless network using Position responsive routing Protocol" in IEEE Symposium on Computers \& Informatics ISCI 2011, Malaysia, pp. 51-55.
[35] Ponnusamy, V., Abdullah, A., and Downe, A. G. (2012). Energy Efficient Routing Protocols in Wireless Sensor Networks: A Survey. Wireless Sensor Networks and Energy Efficiency: Protocols, Routing, and Management, 237-261.

[36] Nurhayati, Sung Hee Choi, and Kyung Oh Lee : A Cluster Based Energy Efficient Location Routing Protocol in Wireless Sensor Network, in International Journal of Computers and Communications, issue 2, Volume 5. 2011. Pp, 67- 74.

[37] Laura Marie Feeney, Martin Nilsson, Article: Investigating the Energy Consumption of a Wireless Network Interface in an Ad Hoc Networking Environment. IEEE Infocom, 2001. 